\title{
Treatment-interval associated effect of irradiation on locoregionally-relapsed ovarian cancer
}

\author{
MOTOAKI SAITO $^{1}$, CHIHIRO KANEHIRA ${ }^{2}$ and SEIJI ISONISHI ${ }^{3}$ \\ Departments of ${ }^{1}$ Obstetrics and Gynecology, ${ }^{2}$ Radiology, Jikei University School of Medicine, Tokyo 105-8461; \\ ${ }^{3}$ Department of Obstetrics and Gynecology, Jikei Daisan Hospital, Tokyo 201-8601, Japan
}

Received April 11, 2014; Accepted May 5, 2014

DOI: $10.3892 / \mathrm{mco} .2014 .316$

\begin{abstract}
Recurrent ovarian cancer following chemotherapy is usually incurable, particularly when the tumor acquires a drug resistance. The present study aimed to define the effect of irradiation on locoregional recurrences and the impact of the factors on the efficacy. The study retrospectively reviewed the clinical records of 61 patients with epithelial ovarian cancer who received irradiation following repeated chemotherapy between 1997 and 2006. A positive-irradiation response was designated as complete response, partial response, minor response or no change (NC). Due to the possible synergistic effect of chemotherapy and irradiation, and the cross-resistance to chemotherapeutic drugs and radiation, the focus was on the treatment break between chemotherapy and radiation, and patients were classified into 3 categories: Category I, $\leq 1$ month; II, 1-6 months; and III, $>6$ months. The effect of irradiation was analyzed in association with histology, treatment break, recurrent site, irradiation dose and chemosensitivity. The post-irradiation survival time was analyzed by the irradiation response and treatment category. The median biological-effective dose was 60.0 Gy (range, 15.6-72.0 Gy). The sites irradiated included nodal recurrence (36), abdominal (six) and pelvic cavity (five cases). Histologically, serous adenocarcinoma was the most common type of the disease (23 cases) compared to mucinous (four), endometrioid (three), and clear-cell types (six cases). The median survival times were 4.5 months in the radiation responders (13 cases) and 15.3 months in the non-responders (37) $(\mathrm{P}=0.004)$. The positive-irradiation response was significantly associated with the treatment break $(\mathrm{P}=0.026)$ and chemosensitivity $(\mathrm{P}=0.007)$. In conclusion, irradiation for recurrent ovarian cancer produced an improved survival benefit when applied to chemoresponsive, locoregional-recurrent tumors immediately following chemotherapy.
\end{abstract}

Correspondence to: Professor Seiji Isonishi, Department of Obstetrics and Gynecology, Jikei Daisan Hospital, 4-11-1 Izumi-Honchou, Komae-shi, Tokyo 201-8601, Japan

E-mail: isonishi@jikei.ac.jp

Key words: ovarian cancer, relapse, irradiation, treatment interval

\section{Introduction}

There is no curative therapy for persistent or recurrent disease in ovarian carcinoma, despite the development of novel chemotherapeutic drugs. Frequently, second- or third-line therapies cannot be performed completely due to the accumulated toxicities, emergence of drug resistance and the terminal declines in performance status.

Radiation therapy has been previously challenged in the treatment of epithelial ovarian tumors. Curative irradiation strategies, including whole abdominal techniques for selected patients with microscopic disease following debulking surgery, have been reported (1-5). Effective palliative irradiation following chemotherapy failure has also been reported, even in the absence of a substantial survival advantage (6-8). However, this treatment became almost obsolete due to the remarkable progression of up-scaled platinum-containing systemic chemotherapies and little or no guideline-based recommendations for the use of irradiation for disseminated tumors. However, more recently, revival of the irradiation has been argued and reported in non-serous ovarian subtypes or locoregionally-recurrent ovarian cancer (9-11).

As the chemotherapeutic drugs used in ovarian cancer and radiation are the same DNA-damaging agent, it is natural that the intrinsic drug-resistant cells could be resistant to radiation. In vitro studies have also suggested the cross-resistance between the platinum agents and irradiation through the overexpression of the ras gene $(12,13)$. By contrast, the radiosensitization effect of the chemotherapeutic drug could be expected when radiation is used concurrently or sequentially similar to the case of cervical cancer $(14,15)$.

The present study reviews the experience of irradiation in patients with recurrent ovarian cancer, who have been repeatedly treated with platinum-based chemotherapy in the Jikei University School of Medicine (Tokyo, Japan). The radiation-effect availability was questioned and the factors impacting the efficacy were examined.

\section{Patients and methods}

Patients. The study is a retrospective analysis of 71 patients evaluated for the relapse of epithelial ovarian cancer between 1997 and 2006. The patients were referred to the Department of Obstetrics and Gynecology by one of the 
four affiliated hospitals of the Jikei University School of Medicine when recurrent-focal lesions developed following second- or third-line treatment. The initial management included exploratory laparotomy, hysterectomy, salpingo-oophorectomy and mostly platinum-containing chemotherapy regimens. Nodal dissection was not routine.

Eligibility was identified by a stepwise process. Metastatic tumors, other than ovarian cancer, and tumors of non-epithelial histology or borderline tumors were excluded. The patients were selected to receive irradiation on a case-by-case basis following a discussion among gynecological and radiological oncologists. The factors that were considered in deciding whether to offer irradiation included the ability to encompass the locoregional disease by the irradiation field, performance status, no ascites and limitations of the other treatment options.

Irradiation was largely abandoned in the group of hospitals after 2004, following the announcement of the consensus statements on the management of ovarian cancer in the 3rd International Gynecologic Cancer Intergroup Ovarian Cancer Consensus Conference (16) and when it was recognized that the combination of platinum and taxane was a highly-active systemic agent. Due to its current infrequent use, the clinical information was acquired from medical records over a decade ago, and certain data were missing due to the paucity of each record. In the study, the focus was on the association between the irradiation efficacy and chemotherapy response, and the treatment break following the completion of chemotherapy up to the initiation of radiation therapy. Therefore, 10 out of 71 patients with brain metastasis were excluded as the blood-brain barrier disabled the evaluation of the chemotherapeutic effect. The study was approved by the Institutional Review Board in Jikei University School of Medicine and conformed to the policies and practices for human subject research.

Chemotherapy. The clinical response to the initial chemotherapy was assessed in 55 patients with a measurable disease based on the classical WHO criteria (17). A complete response (CR) was defined as the disappearance of all the clinical evidence of the malignant disease, and a partial or minor response (PR or MR) was a $>50$ or $25 \%$ decrease in the size of the clinically measurable disease, respectively. In the study, a positive response was designated as CR, PR or NC.

Irradiation. For the prognostic-significance test of the radiation dose, the dose fractionation schemes were converted to a biologically-effective dose (BED) using the following formula: $\mathrm{BED}=$ total dose $[1+$ fractional dose $/(\alpha / \beta)], \alpha / \beta=10 \mathrm{~Gy}$.

The patients were followed up jointly by gynecological and radiation oncologists. The response data were extracted from all the records of these two oncological disciplines. Due to the paucity of data, the exact response rate was not calculated in the present retrospective study. The responders were designated as the patients who showed $\geq$ no change (NC) of documented recurrent tumor determined by computerized axial tomography, magnetic resonance imaging or ultrasonography approximately within 1 month after completion of irradiation. As the radiation effect was often apparent 3-6 months after (18), the response criteria in the study included the cases of NC. The toxicity by irradiation was not assessed since the detailed clinical records were not available.

Chemotherapy-irradiation break. Theoretically, chemotherapy and radiotherapy could have a synergistic effect and the chemotherapy may function as a radiosensitizer, similarly to the case of concurrent chemotherapy in cervical cancer (14). By contrast, the cancer cells surviving the previous platinum-containing chemotherapy could acquire and maintain the platinum resistance within 6 months after chemotherapy according to the Gynecologic Oncology Group (GOG) criteria and the platinum resistance may be cross-resistant to ionizing radiation as well. On the bases of this cellular biology, the patients were categorized into three groups by the treatment breaks prior to the initiation of irradiation: Category I, $\leq 1$ month; II, 1-6 months; and III, >6 months.

Data were collected for age, histological type, recurrent site, prior chemotherapeutic treatments, total dose, BED, the treatment break between the last chemotherapy and irradiation, response to irradiation as well as previous chemotherapy and post-irradiation survival time.

Statistical analysis. The overall survival time was measured from the date of the initiation of irradiation. The duration of the survival time was measured up to the date of mortality or the date of the last contact if the patient remained. A total of 46 eligible cases were included in the survival-time analysis unless otherwise specified. All the causes of mortality were used to calculate the survival time, and the estimates of the cumulative proportion surviving were based on Kaplan-Meier procedures (19). For the post-irradiation survival time, the Cox proportional-hazards regression model was used to estimate the treatment relative hazards (20). Pearson's $\chi^{2}$ test was used to test the independence of the response and treatment (21). Data were analyzed using Stata software (StataCorp, College Station, TX, USA). The primary outcome was considered to indicate a statistically significant difference when $\mathrm{P}<0.05$.

\section{Results}

The demographics and disease characteristics of the patients are summarized in Table I. The total number of each factor was not exactly 61 due to certain data being missing. Prior to irradiation, the patients received a median of 12 chemotherapy courses (range, 5-14), and the majority were composed of the platinum-taxane combination. The median BED was 60.0 Gy (range, 15.6-72.0 Gy). The sites irradiated included nodal recurrence (36 cases), abdominal (six cases), and pelvic cavity (five cases). The majority of patients did not complain of tumor-associated symptoms, however, the most common symptoms of pain and vaginal bleeding were caused by disease in the abdominal (six cases) or pelvic cavity (five cases). Histologically, serous adenocarcinoma was the most common type of the disease (23 cases, $38 \%$ ) compared to mucinous (four cases, 7\%), endometrioid (three cases, 5\%), and clear-cell types (six cases, 10\%).

Post-irradiation survival time. Figs. 1-3 show the post-irradiation survival curves for all the eligible females by the category, radiation response and drug sensitivity. The median 
Table I. Patient profiles.

\begin{tabular}{|c|c|c|}
\hline & $\mathrm{n}(\%)$ & Median (min-max) \\
\hline Age, year & 58 & $54.0(26.0-71.0)$ \\
\hline Total dose, Gy & 58 & $47.7(12.5-60.0)$ \\
\hline $\mathrm{BED}^{\mathrm{a}}$, Gy & 55 & $60.0(15.6-72.0)$ \\
\hline Survival $^{\mathrm{b}}$, Mo & 50 & $11.5(0.5-82.4)$ \\
\hline Interval $^{\mathrm{c}}, \mathrm{Mo}$ & 53 & $3.2(0.0-63.8)$ \\
\hline Site & 61 & \\
\hline $\begin{array}{l}\text { Lymph node } \\
\text { (Virchow) }\end{array}$ & $19(31.1)$ & \\
\hline Lymph node & $17(27.9)$ & \\
\hline Abdomen & $6(9.8)$ & \\
\hline Pelvis & $5(8.2)$ & \\
\hline Mediastinum & $4(6.6)$ & \\
\hline Lung & $3(4.9)$ & \\
\hline Others & $4(6.6)$ & \\
\hline Unclear & $3(4.9)$ & \\
\hline Histology & 61 & \\
\hline Serous & $23(37.7)$ & \\
\hline Mucinous & $4(6.6)$ & \\
\hline Endometrioid & $3(4.9)$ & \\
\hline Clear cell & $6(9.8)$ & \\
\hline $\begin{array}{l}\text { Poorly } \\
\text { differentiated }\end{array}$ & $3(4.9)$ & \\
\hline Adenocarcinoma & $10(16.4)$ & \\
\hline Others $^{\mathrm{d}}$ & $4(6.6)$ & \\
\hline Unclear & $8(13.1)$ & \\
\hline Chemotherapy & 61 & \\
\hline Platinum & $57(93.4)$ & \\
\hline Taxane & $42(68.9)$ & \\
\hline Irinotecan & $5(8.2)$ & \\
\hline Cytoxan & $5(8.2)$ & \\
\hline Doxorubicin & $18(28.5)$ & \\
\hline
\end{tabular}

${ }^{\mathrm{a} B i o l o g i c a l}$ effective dose $=$ total dose $/(1+$ fractional dose $/ 10) ;{ }^{\mathrm{b}} \mathrm{me}-$ dian survival following irradiation; 'interval between chemotherapy and irradiation; ${ }^{\mathrm{d}}$ carcinosarcoma (two cases), squamous cell carcinoma (one case) and small cell carcinoma (one case).

duration of follow-up for females who remained at the last contact point was 16 months (range, 1-82 months). There was no statistically significant difference among the treatment categories (Fig. 1). However, the patients whose disease responded to the radiation therapy had a higher post-irradiation survival rate than patients with radiation-non-responded disease. The median survival time in the responded group was 16 months, and in the non-responded group it was 2 months [hazard ratio (HR), $0.39 ; \mathrm{P}=0.013 ; 95 \%$ confidence interval (CI), 0.19-0.82] (Fig. 2). Similarly, patients whose disease responded to the initial chemotherapy had a higher survival rate than patients without a response. The median survival time in the chemo-responded group was 17 months, and in

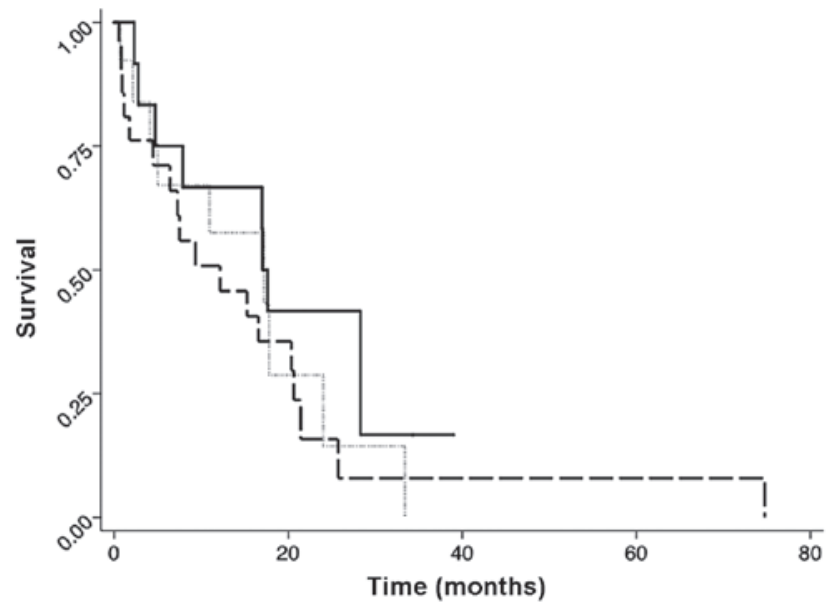

Figure 1. Kaplan-Meier estimates of the post-irradiation survival time by the treatment category. Solid line; category I, dashed line; category II, dotted line; category III,. No statistically significant difference was noted among the treatment categories.

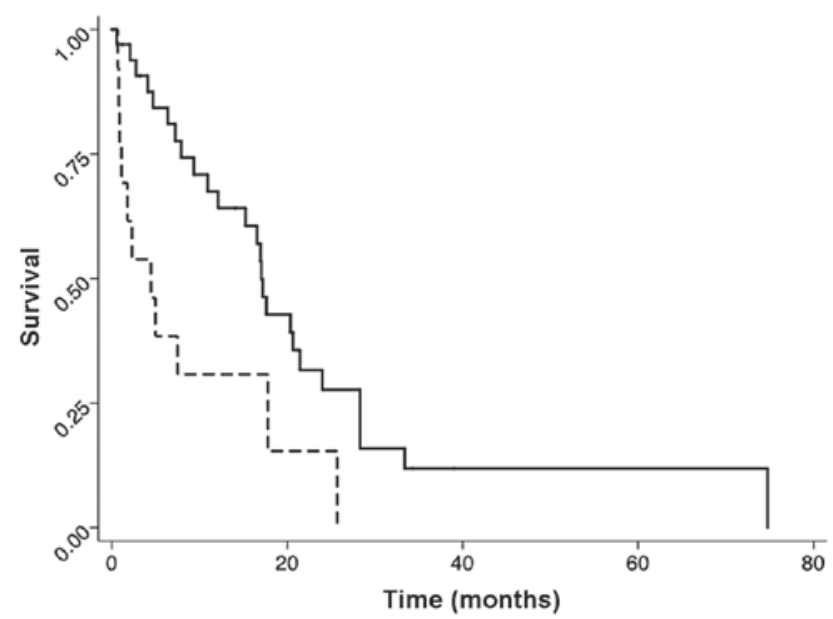

Figure 2. Kaplan-Meier estimates of post-irradiation survival time by the radiation response. The median survival times in the responded (solid line) and non-responded (dashed line) groups were 16 and 2 months, respectively [hazard ratio (HR), 0.39; $\mathrm{P}=0.013 ; 95 \%$ confidence interval (CI), 0.19-0.82].

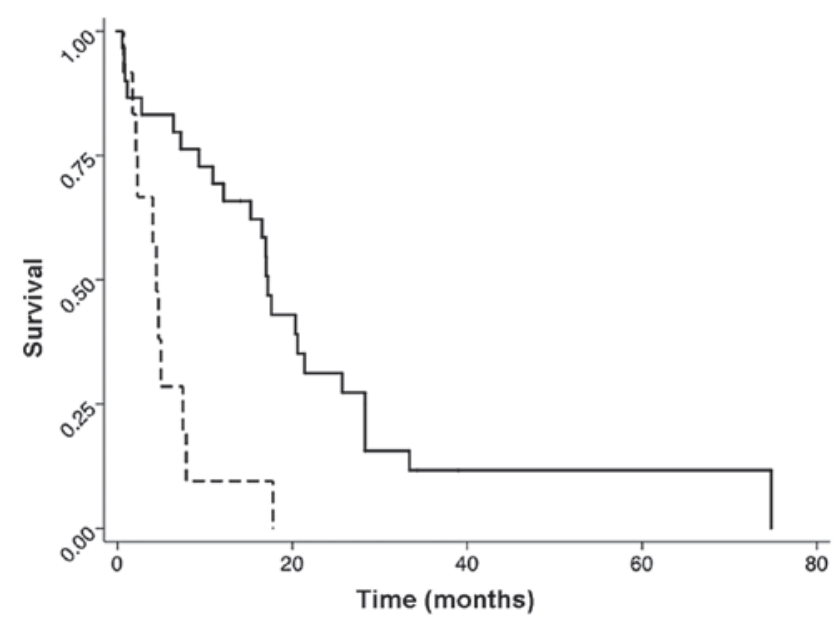

Figure 3. Kaplan-Meier estimates of post-irradiation survival time by the chemotherapy response. The median survival times in the chemo-responded and non-responded groups were 17 and 4 months, respectively [hazard ratio (HR), $0.23 ; \mathrm{P}=0.001 ; 95 \%$ confidence interval $(\mathrm{CI}), 0.10-0.54]$. 
Table II. Association of the clinicopathological variables to the radiation responsibility.

\begin{tabular}{|c|c|c|c|c|c|}
\hline \multirow[b]{2}{*}{ Variable (n) } & \multirow[b]{2}{*}{ Category } & \multicolumn{3}{|c|}{ Response } & \multirow{2}{*}{$\begin{array}{c}\text { Pearson's } \\
\chi^{2}\end{array}$} \\
\hline & &,$- \mathrm{n}$ &,$+ \mathrm{n}$ & $\%$ & \\
\hline \multirow[t]{2}{*}{ Histology (61) } & Serous & 3 & 19 & 86.4 & \\
\hline & Non-serous & 12 & 27 & 69.2 & 0.136 \\
\hline \multicolumn{6}{|l|}{ Treatment break (53) } \\
\hline \multicolumn{6}{|l|}{ Category $^{\mathrm{a}}$} \\
\hline I vs. II & $\mathrm{I}$ & 1 & 14 & 93.3 & 0.026 \\
\hline II vs. III & II & 9 & 14 & 60.9 & 0.357 \\
\hline I vs. III & III & 4 & 11 & 75.0 & 0.165 \\
\hline \multirow[t]{2}{*}{ Site $(61)$} & Lymph node & 6 & 30 & 83.3 & \\
\hline & Other & 9 & 16 & 64.0 & 0.085 \\
\hline \multirow[t]{2}{*}{$\mathrm{BED}^{\mathrm{b}}$, Gy (58) } & $\leq 60$ & 8 & 20 & 71.4 & \\
\hline & $>60$ & 7 & 23 & 76.7 & 0.649 \\
\hline \multirow[t]{2}{*}{ Chemo response (54) } & - & 7 & 6 & 46.2 & \\
\hline & + & 6 & 35 & 85.4 & 0.007 \\
\hline
\end{tabular}

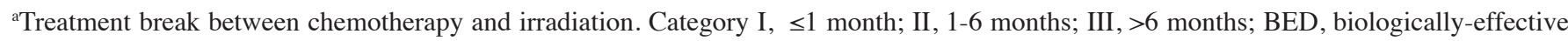
dose. ${ }^{b} \mathrm{BED}=$ total dose $/(1+$ fractional dose $/ 10)$.

the non-responded group it was 4 months $(\mathrm{HR}, 0.23 ; \mathrm{P}=0.001$; 95\% CI, 0.10-0.54) (Fig. 3).

Clinicopathological variables. Table II shows the variables that were associated with the radiation effect. The histotype of serous adenocarcinoma was not associated with the radiation responsibility compared to the cluster of other histotypes. Re-clustering clear cell, mucinous and endometrioid into one group compared to serous or other types did not alter the result. However, when the radiation-sensitivity of serous type (23 cases) was compared to the cluster of clear cell, endometrioid and mucinous types (13 cases), serous type was more sensitive $(\mathrm{P}=0.033$, data not shown $)$.

Treatment break. A treatment break of $\leq 1$ month (category I) is an important factor associated with the positive-radiation response compared to category II (93 vs. $61 \%$; $\mathrm{P}=0.026)$. The radiation response in category III ( $>6$ months) appeared to be improved compared to category II (1-6 months) (75 vs. 61\%; $\mathrm{P}=0.357$ ), however this is not significant.

The comparison of the reported site of relapse for irradiation and the amount of BED did not show any associations in the rate of the radiation response. The patients whose disease responded to the initial chemotherapy had a higher rate of radiation responsibility compared to patients with chemotherapy non-responded disease ( 85 vs. $46 \%$; $\mathrm{P}=0.007$ ).

\section{Discussion}

The results of the present study provide strong evidence that irradiation can play an important role in curative management of locoregionally-confined recurrent or persistent ovarian cancer. The foundation of the data is the improved post-irradiation survival rate in the patients with radiation-responsive recurrent disease compared to those with non-responsive disease, and the clear association between the radiation effect with the treatment break or chemo-responsibility.

Several points should be discussed with regards to the study. First, the radiation-response criteria designated in the study was not so strict that numerous cases were categorized as the radiation-responsive group. This is due to the physicians being unfamiliar with the response criteria in the solid tumor at the time and due to various missing imaging data that did not allow for the second chance of re-evaluation. Similarly, the positive-chemotherapy response criteria in the study covered a broad range of response, including $\mathrm{NC}$, resulting in a large number of responsive cases with the same reason as the radiation-responsibility criteria. The present study demonstrated that the chemo-responsibility associated well with the outcome of the chemotherapy-responded patients having a longer post-irradiation survival time than non-responded patients (Fig. 3), and it was also well associated with the radiation-responsibility (Table II). Similar findings were also reported in two other studies $(11,22)$, showing that the platinum-sensitivity associated with the prognostic benefit in locoregionally-recurrent ovarian cancer. In the present study, 93\% of the patients received platinum-containing chemotherapy, indicating that chemo-sensitivity in the study could be translated into platinum-sensitivity.

With respect to the chemo- or radiation-sensitivity, the second important issue was the histological type-associated radiation responsibility. The data from the present study and two other studies $(11,23)$ that contained a large number of serous carcinoma, showed an apparent prognostic or symptomatic benefit from irradiation. However, Swenerton et al (9) 
reported that the use of adjuvant radiation following chemotherapy in microscopic disease showed an incremental survival benefit for the non-serous carcinoma, including clear cell, endometrioid and mucinous adenocarcinomas, and not for the serous type $(9,10)$. In the present study, the irradiation was used for recurrent, macroscopic and locoregional diseases, and radiation was used as field-involved irradiation with a median dose of 48 Gy. However, Swenerton et al (9) used radiation for minimal, and usually disseminated, residual disease as adjuvant whole abdominal irradiation, with 23-28 Gy. As the present data contained a large number of unclassified or unclear histotypes, the study is not adequate in showing the histotype-specific radiation-sensitivity, however, the aforementioned inequality of the targeting tumor, as well as the total dose, makes it hard to conclude the histotype-specific radiation sensitivity.

Finally, the treatment break associated with the radiation-response should be noted. The present data showed that irradiation after $\leq 1$ month break (category I) was well associated with the radiation-response compared to 1-6 months (category II), despite the lack of a survival benefit. In category I, when the tumor cells were not intrinsically resistant and therefore the tumor responded to primary chemotherapy, the preceding chemotherapy could have the potential to augment the effects of radiation, similar to the case of concurrent chemoradiation with cisplatin in cervical cancer (14). A similar effect was also noted when the radiation was used sequentially following paclitaxel plus carboplatin (15). In the study, sequential radiation was initiated 2-3 weeks after the completion of the last chemotherapy cycle, but the interval was allowed to be extended and this is exactly the same as category I in the present study. The present study demonstrated a poorer locoregional control when irradiation was delayed to 1-6 months after the completion of chemotherapy, but the response appeared to be recovered slightly when the time break was extended to $>6$ months. This could be attributable to the possible acquired cross-resistance to chemotherapy and radiation therapy for the patients with a treatment break of 1-6 months, which is consistent with the treatment-free interval of absolute platinum resistant in the GOG criteria.

The present study has several limitations, including a comprising heterogeneous study population, lack of toxicity data and tumor size. However, the study suggests that irradiation could yield a survival benefit when the treatment is applied to chemosensitive, locoregionally-recurrent ovarian cancer and when the treatment is initiated $\leq 1$ month after the preceding chemotherapy.

\section{Acknowledgements}

The authors would like to thank Mr. G. Rupelle, lecturer of Jikei Kashiwa Hospital (Tokyo, Japan), for the critical review of the present study.

\section{References}

1. Dembo AJ: Radiotherapeutic management of ovarian cancer Semin Oncol 11: 238-250, 1984.
2. Einhorn N, Tropé C, Ridderheim M, Boman K, Sorbe B and Cavallin-Ståhl E: A systematic overview of radiation therapy effects in ovarian cancer. Acta Oncol 42: 562-566, 2003.

3. Thomas GM and Dembo AJ: Integrating radiation therapy into the management of ovarian cancer. Cancer 71 (Suppl 4): 1710-1718, 1993.

4. Martinez A, Schray MF,Howes AE and Bagshaw MA: Postoperative radiation therapy for epithelial ovarian cancer: the curative role based on a 24-year experience. J Clin Oncol 3: 901-911, 1985.

5. Cardenes $\mathrm{H}$ and Randall ME: Integrating radiation therapy in the curative management of ovarian cancer: current issues and future directions. Semin Radiat Oncol 10: 61-70, 2000.

6. Albuquerque KV, Singla R, Potkul RK, et al: Impact of tumor volume-directed involved field radiation therapy integrated in the management of recurrent ovarian cancer. Gynecol Oncol 96: 701-704, 2005

7. May LF, Belinson JL and Roland TA: Palliative benefit of radiation therapy in advanced ovarian cancer. Gynecol Oncol 37: 408-411, 1990 .

8. Fujiwara K, Suzuki S, Yoden E, Ishikawa H, Imajo Y and Kohno I: Local radiation therapy for localized relapsed or refractory ovarian cancer patients with or without symptoms after chemotherapy. Int J Gynecol Cancer 12: 250-256, 2002.

9. Swenerton KD, Santos JL, Gilks CB, et al: Histotype predicts the curative potential of radiotherapy: the example of ovarian cancers. Ann Oncol 22: 341-347, 2011.

10. Thomas G: Revisiting the role of radiation treatment for non-serous subtypes of epithelial ovarian cancer. American Society of Clinical Oncology Educational Book/ASCO. American Society of Clinical Oncology Meeting e205, Toronto, 2013.

11. Brown AP, Jhingran J, Klopp AH, Schmeler KM, Ramirez PT and Eifel PJ: Involved-field radiation therapy for locoregionally recurrent ovarian cancer. Gynecol Oncol 130: 300-305, 2013

12. Sklar MD: Increased resistance to cis-diamminedichloroplatinum(II) in NIH 3T3 cells transformed by ras oncogenes. Cancer Res 48: 793-797, 1988.

13. Sklar MD: The ras oncogenes increase the intrinsic resistance of NIH 3T3 cells to ionizing radiation. Science 239: 645-647, 1988.

14. Rose PG, Bundy BN, Watkins EB, et al: Concurrent cisplatin-based radiotherapy and chemotherapy for locally advanced cervical cancer. N Engl J Med 340: 1144-1153, 1999.

15. Sehouli J, Runnebaum IB, Fotopoulou C, et al: A randomized phase III adjuvant study in high-risk cervical cancer: simultaneous radiochemotherapy with cisplatin $(\mathrm{S}-\mathrm{RC})$ versus systemic paclitaxel and carboplatin followed by percutaneous radiation (PC-R): a NOGGO-AGO Intergroup Study. Ann Oncol 23: 2259-2264, 2012.

16. Quinn M, Avall-Lundqvist E, du Bois A, et al: History, scope and methodology of the 3rd international consensus workshop on ovarian cancer 2004. Ann Oncol 16 (Suppl 8): viii5-viii6, 2005.

17. WHO Handbook for Reporting Results of Cancer Treatment. WHO Offset Publication no. 48, World Health Organization, Geneva, 1979.

18. Mayr NA, Taoka T, Yuh WT, Denning LM, Zhen WK, Paulino AC, et al: Method and timing of tumor volume measurement for outcome prediction in cervical cancer using magnetic resonance imaging. Int J Radiat Oncol Biol Phys 52: 14-22, 2002

19. Kaplan EL and Meier P: Nonparametric estimation from incomplete observations. J Am Stat Assoc 53: 457-481, 1958.

20. Cox DR: Regression models and life-tables (with discussion). J Royal Stat Soc, Series B 34: 187-220, 1972.

21. Agresti A: Categorical Data Analysis. John Wiley \& Sons, New York, NY, 1990.

22. Lee SW, Park SM, Kim YM, Kim YS, Choi EK, Kim DY, et al: Radiation therapy is a treatment to be considered for recurrent epithelial ovarian cancer after chemotherapy. Tumori 97: 590-595, 2011.

23. Choan E, Quon M, Gallant V and Samant R: Effective palliative radiotherapy for symptomatic recurrent or residual ovarian cancer. Gynecol Oncol 102: 204-209, 2006. 